University of Wollongong

Research Online

Australian Institute for Innovative Materials -

Papers

Australian Institute for Innovative Materials

$1-1-2019$

\title{
Challenges and prospects in the catalysis of electroreduction of nitrogen to ammonia
}

Bryan H. R Suryanto

Monash University

Hoang-Long Du

Monash University

Dabin Wang

Monash University

Jun Chen

University of Wollongong, junc@uow.edu.au

Alexandr N. Simonov

Monash University, alexandr.simonov@monash.edi

See next page for additional authors

Follow this and additional works at: https://ro.uow.edu.au/aiimpapers

Part of the Engineering Commons, and the Physical Sciences and Mathematics Commons

Research Online is the open access institutional repository for the University of Wollongong. For further information contact the UOW Library: research-pubs@uow.edu.au 


\title{
Challenges and prospects in the catalysis of electroreduction of nitrogen to ammonia
}

\author{
Abstract \\ Ammonia is a widely produced chemical that is the basis of most fertilisers. However, it is currently \\ derived from fossil fuels and there is an urgent need to develop sustainable approaches to its production. \\ Ammonia is also being considered as a renewable energy carrier, allowing efficient storage and \\ transportation of renewables. For these reasons, the electrochemical nitrogen reduction reaction (NRR) is \\ currently being intensely investigated as the basis for future mass production of ammonia from \\ renewables. This Perspective critiques current steps and miss-steps towards this important goal in terms \\ of experimental methodology and catalyst selection, proposing a protocol for rigorous experimentation. \\ We discuss the issue of catalyst selectivity and the approaches to promoting the NRR over $\mathrm{H} 2$ \\ production. Finally, we translate these mechanistic discussions, and the key metrics being pursued in the \\ field, into the bigger picture of ammonia production by other sustainable processes, discussing \\ benchmarks by which NRR progress can be assessed.

\section{Disciplines} \\ Engineering | Physical Sciences and Mathematics

\section{Publication Details} \\ Suryanto, B. H. R., Du, H., Wang, D., Chen, J., Simonov, A. N. \& MacFarlane, D. R. (2019). Challenges and \\ prospects in the catalysis of electroreduction of nitrogen to ammonia. Nature Catalysis, 2 (4), 290-296.
}

\section{Authors}

Bryan H. R Suryanto, Hoang-Long Du, Dabin Wang, Jun Chen, Alexandr N. Simonov, and Douglas R. MacFarlane 


\title{
Challenges and Prospects in the Catalysis of Electroreduction of Nitrogen to Ammonia
}

\author{
Bryan H. R. Suryanto ${ }^{1}$, Hoang Long Du ${ }^{1,2}$, Dabin Wang ${ }^{1}$, Jun Chen ${ }^{3}$, Alexandr N. Simonov ${ }^{1,2}$ and \\ Douglas R. MacFarlane ${ }^{1,2, *}$ \\ ${ }^{1}$ School of Chemistry, Faculty of Science, Monash University, VIC 3800, Australia \\ ${ }^{2}$ ARC Centre of Excellence for Electromaterials Science, Monash University, VIC 3800, Australia \\ ${ }^{3}$ ARC Centre of Excellence for Electromaterials Science, University of Wollongong, NSW 2500,
}

Australia

Correspondinge-mail: douglas.macfarlane@monash.edu

\begin{abstract}
Ammonia is a widely produced chemical that is the basis of most fertilisers. However, it is currently derived from fossil fuels and there is an urgent need to develop sustainable approaches to its production. Ammonia is also being considered as a renewable energy carrier, allowing efficient storage and transportation of renewables. For these reasons, the electrochemical nitrogen reduction reaction (NRR) is currently being intensely investigated as the basis for future mass-production of ammonia from renewables. This Perspective critiques current steps and miss-steps towards this important goal in terms of experimental methodology and catalyst selection, proposing a protocol for rigorous experimentation. We discuss the issue of catalyst selectivity and the approaches to promoting the $\mathrm{NRR}$ over $\mathrm{H}_{2}$ production. Finally, we translate these mechanistic discussions, and the key metrics being pursued in the field, into the bigger picture of ammonia production by other sustainable processes, discussing benchmarks by which NRR progress can be assessed.
\end{abstract}

\section{Introduction}

Ammonia synthesis from molecular nitrogen and hydrogen was one of the greatest inventions of the $20^{\text {th }}$ century. This century-old Haber-Bosch process is today the source of most of the world's ammonia, contributing $90 \%$ of annual production ${ }^{1}$. Ammonia, and its derivatives including urea, is a vitally important component of fertilisers ${ }^{2}$. It is estimated that global food production could only support a fraction of today's world population without the use of artificial fertilisers from the HaberBosch process ${ }^{3,4}$. However, the prospect of continued and growing use of this process for future supplies of ammonia creates serious environmental concerns ${ }^{5}$ as fossil fuels (mainly natural gas) are the main source of the $\mathrm{H}_{2}$ precursor. Exacerbating this issue, the sluggish kinetics of the reaction between $\mathrm{N}_{2}$ and $\mathrm{H}_{2}$ requires elevated temperatures $\left(\mathrm{ca} 500{ }^{\circ} \mathrm{C}\right)$ and pressures ( $\left.>200 \mathrm{~atm}\right)$, rendering the overall process highly energetically demanding ${ }^{6}$. As a result, approximately $2 \%$ of anthropogenic energy is consumed, and $400 \mathrm{Mt}$ of $\mathrm{CO}_{2}$ is released, annually, to sustain ammonia production at the level required to satisfy current demand ${ }^{1,7}$.

Electrochemical methods offer the hope of directly converting renewable electricity into chemicals and chemical energy carriers. The latter context reflects a growing recognition that there is enormous capacity to generate renewable energy at very low cost in certain regions of the world, for example northwestern Australia, but that the remote locations involved require some means of transporting the energy to distant, often overseas, centres of population. Ammonia is increasingly recognised as a highly viable chemical energy carrier, with a well-known transportation technology on bulk 
carriers and pipelines. Recent reports demonstrate methods of converting it at point of use, if required, into hydrogen ${ }^{8}$. As a result, interest in the electrochemical nitrogen reduction reaction (NRR) to ammonia under ambient conditions, has increased markedly in recent years with a focus on improving the two key NRR performance metrics: (i) the ammonia formation selectivity (also known as Faradaic Efficiency, FE) and (ii) the yield rate. From a thermodynamic point of view, the NRR should benefit from a higher energy efficiency, by about 20\%, in contrast to the Haber-Bosch process ${ }^{9}$. Moreover, the NRR approach would also enable the decentralised production of ammonia that may ultimately support distributed fertiliser production.

However, efficient NRR has been proven to be extremely challenging to achieve in practice, underscoring the fact that $\mathrm{N}_{2}$ is a highly stable, non-polarisable molecule ${ }^{10,11}$. This typically shifts the potentials needed to reduce dinitrogen into the region where proton reduction to hydrogen becomes facile as a competing reaction. Multiple strategies have been explored to minimise the occurrence of the hydrogen evolution reaction (HER), both from a catalyst and an electrolyte perspective. Nonetheless, selectivity remains quite low in aqueous solution based NRR, typically not more than $15 \%$. On the other hand, strategies to suppress the competing HER have demonstrated the potential to achieve FEs of $35 \%{ }^{12}$ and even above $60 \%^{13}$.

This perspective article discusses recent progress in the field of ammonia electrosynthesis and highlights a number of significant issues and challenges that are arising in this research. Reports published in the past 3 years have described dramatic improvements in efficiency and rate. However, these improvements should be treated with caution, particularly in identifying optimum electrocatalysts, and understanding the possible NRR mechanisms involved, due to issues regarding accurate detection of $\mathrm{NH}_{3}$ from $\mathrm{N}_{2}$ reduction, as opposed to other extraneous sources. This Proof of NRR issue is one of the main focal points of this perspective and we discuss this in some detail, proposing definitive control experiments and methodologies. With this literature reliability issue in mind, we then offer a perspective of catalyst selectivity and reaction energetics from a theoretical point of view. We also compare the current state of the field with alternative approaches to NRR that provide important practical benchmarks in terms of performance. Space limitations restrict our discussion to an overview of some of the issues and instead we provide key references to act as an entry point to some of these topics for the interested reader.

\section{Proof of NRR - Challenges in detection methods and control experiments}

The fundamental issue in much of the recent research on NRR is that the quantities of ammonia produced are very small. Typical experimental yields are in the range of $10-1000 \mathrm{nmol}$. Unfortunately, ammonia is ubiquitous in the laboratory environment and is a common contaminant in chemicals, especially gases. It is important to note that the level of contamination in any material or chemical is highly dependent on its manufacturer, storage and handling. Being a volatile gas it also tends to accumulate on common glassware surfaces or into aqueous solutions over time ${ }^{14} 15,16$, in particular in a laboratory environment, or building, where ammonia is used and/or produced. Atmospheric ammonia levels vary with season (higher in summer) and regionally, depending on the intensity of agricultural and industrial activity ${ }^{17}$. Levels between 0.04 and $0.2 \mu \mathrm{mol} \mathrm{m}{ }^{-3}$ are routinely observed around the world ${ }^{17}$. However, in regions having impaired air quality atmospheric ammonia can reach much higher levels, for example recent observations of $>6 \mu \mathrm{mol} \mathrm{m}^{-3}$ in Beijing ${ }^{18}$; such airborne sources of ammonia may represent a significant experimental issue ${ }^{16}$. The challenge for a conscientious laboratory, therefore, is to unambiguously demonstrate that the ammonia determined in an NRR experiment has been substantially produced by electrochemical reduction of dinitrogen and not from some other, exogenous source. The photochemical nitrogen fixation field has been dogged by these issues for more than 30 years and its literature is awash with studies since proven to be incorrect or not reproducible in other laboratories due to the measured $\mathrm{NH}_{3}$ having been produced or introduced from sources of $\mathrm{N}$ other than by the reduction of supplied $\mathrm{N}_{2}$ gas (see Boucher et al. ${ }^{19}$ and the many references mentioned therein). Research on biological fixation of nitrogen, in which the biomaterials involved provide a clear source of potential contamination, has long recognised the need for rigorous confirmatory experiments. The NRR field has much to learn 
from this history - or much to ignore at its peril, one of the key learnings being that ${ }^{15} \mathrm{~N}_{2}$ experiments are a vital component of research results, because exogenous sources of ammonia are almost impossible to avoid. Clearly no-one wants to see a repeat of the pathological science of the Cold Fusion ${ }^{20}$ and Polywater ${ }^{21}$ sagas, in which (i) lack of completely adequate experimental proof and (ii) measurements that were on the edge of detection limits, produced masses of research activity that was ultimately proven to be invalid. A secondary phenomenon of both of these unfortunate episodes (and one that young researchers entering the NRR field should be mindful of) was the science bandwagon effect by which large numbers of researchers jumped into the field after the first reports, unquestioningly carried out experiments that were only minor variations of the originals, failed to challenge the methodology or reasoning, and eventually were carried along into the ultimate crash.

There are certainly numerous N-containing materials, catalysts, electrolyte solvents and gases currently in use in the NRR field that can potentially contribute to the measured amount of ammonia. Oxidised forms of nitrogen (hereinafter, $\mathrm{NO}_{\mathrm{x}}$ ) including anions in chemicals/solvents and nitrogen oxides in gas supplies are especially troublesome, as these are very readily reducible to ammonia. The $\mathrm{NO}_{\mathrm{x}}$ in gas supplies is of particular concern as this exogenous source is often supplied continuously in long-running tests, so that even the observation of steady production of $\mathrm{NH}_{3}$ over extended periods is not an unambiguous proof of NRR. Moreover, $\mathrm{NO}_{\mathrm{x}}$ has also been recognised as a common labware contaminant. Ishibashi et al..$^{22}$ and Makela et al. ${ }^{23}$ have observed $\mu$ mol amounts of $\mathrm{NO}_{3}{ }^{-}$and $\mathrm{NO}_{2}{ }^{-}$contaminants being acquired from laboratory gloves and glassware, despite multiple rinsing efforts. A related problem arises whenever there are reducible $\mathrm{N}$-species, or nitrides, or other reducing agents present in the electrode or electrolyte materials. Viewed from a catalysis point of view it is obviously necessary that the $\mathrm{NH}_{3}$ produced by the NRR process exhibit a turnover number (TON) of at least 10 with respect to each of these materials. Only then is the electrocatalytic process reliably demonstrated. To date, in the literature this is rarely the case, because it requires very long-running tests to achieve this condition.

These issues generate the need for rigorous control protocols and confirmatory experimental methods. Preferably these should be widely agreed to in the field such that researchers can be confident that their methodology is sufficient to be accepted. This is emergent, but there are many recent reports describing electrocatalysts that contain $\mathrm{N}$-species, or that were prepared from $\mathrm{N}$ containing precursors, in which the source of the $\mathrm{NH}_{3}$ remains unproven due to insufficient confirmation. This creates an uncertainty about literature data that represents a major constraint on progress. We believe that the paper by Greenlee et al. ${ }^{24}$ represents an important reference point for the field that clearly sets out the experimental methodologies, the control experiments needed and the confirmatory experiments that must be carried out; in particular, ${ }^{15} \mathrm{~N}_{2}$ experiments.

Here, drawing on Greenlee et al. ${ }^{24}$, and adding perspectives from our own experiences, we summarise the protocols that should be followed as necessary and sufficient for Proof of NRR. Where we have added significantly to the recommendations of Greenlee et al, we do so in italics. Figure 1 summarises these steps. Further details are discussed in that paper and the carefully selected references that they highlight as describing rigorous experimental design in useful detail. Many of the methodologies for these tests and controls can be found in Zhou et al. ${ }^{13}$. In the following, we refer to background $\mathrm{NH}_{3}$ that is present or produced from exogenous sources as $\mathrm{x}-\mathrm{NH}_{3}$.

Gas Purification. Gases (Ar, ${ }^{14} \mathrm{~N}_{2}$ and ${ }^{15} \mathrm{~N}_{2}$ ) need to be scrubbed to remove $\mathrm{x}-\mathrm{NH}_{3}$ and $\mathrm{NO}_{\mathrm{x}}$ contaminants and the level of such species (at a relevant limit of detection) demonstrated via ion chromatography, or equivalent, in control samples. $\mathrm{NO}, \mathrm{NO}_{2}, \mathrm{~N}_{2} \mathrm{O}$ are common low-level pollutants in the environment ${ }^{22}$, both in the air and easily dissolved in water to form anions that are readily reduced electrochemically to $\mathrm{NH}_{3}$. For example, gold, silver copper and platinum have all been recognised as excellent electrocatalysts for nitrate/nitrite reduction ${ }^{25-28}$. It is important to appreciate that the impact of $\mathrm{NO}_{\mathrm{x}}$ contamination in the $\mathrm{N}_{2}$ gas supply on apparent $\mathrm{NH}_{3}$ yield is not independently observable in electrochemical experiments and cannot be removed by any background subtraction. It therefore must be quantified for relevant volumes of gas delivered and its potential impact on the reported $\mathrm{NH}_{3}$ yields discussed. 
Control, open-circuit measurements of $\mathrm{x}-\mathrm{NH}_{3}$ in the electrolyte and trap solutions without and with working electrode present, after passage of each gas used and for relevant experimental periods, should be determined and assessed for adequacy in the context of the NRR measurements. [x- $\left.\mathrm{NH}_{3}\right]$ from the $\mathrm{N}_{2}$ supply should be at or below the level observed from the Ar supply. If not, further scrubbing is needed.

Argon control electrochemical measurements at each measured potential should be carried out and presented. Since ammonia can accumulate on surfaces over time, it is important that these be carried out immediately prior to each main NRR experiment and for the same periods of time. These should also be shown as background currents on electrochemical data plots and the measured $\mathrm{x}-\mathrm{NH}_{3}$ amount must be subtracted from the reported $\mathrm{NRR} \mathrm{NH}_{3}$ yields. This is particularly important for long-running tests as the measured yield may be, at least in part, due to the gas or other contamination issues.

$\mathrm{N}$-containing materials. In the case of electrocatalysts which involve $\mathrm{N}$-containing active sites or that have been prepared using nitrogen containing compounds (e.g. nitrate salts in Hummer's method $^{29}$ ) or solvents, at any stage, the Ar control at operating potentials may produce a variable amount of background $\mathrm{x}-\mathrm{NH}_{3}$. We recommend carrying out the Ar control experiments repeatedly over varying periods of resting and electrolysis times in order to qualify a material for further study. 


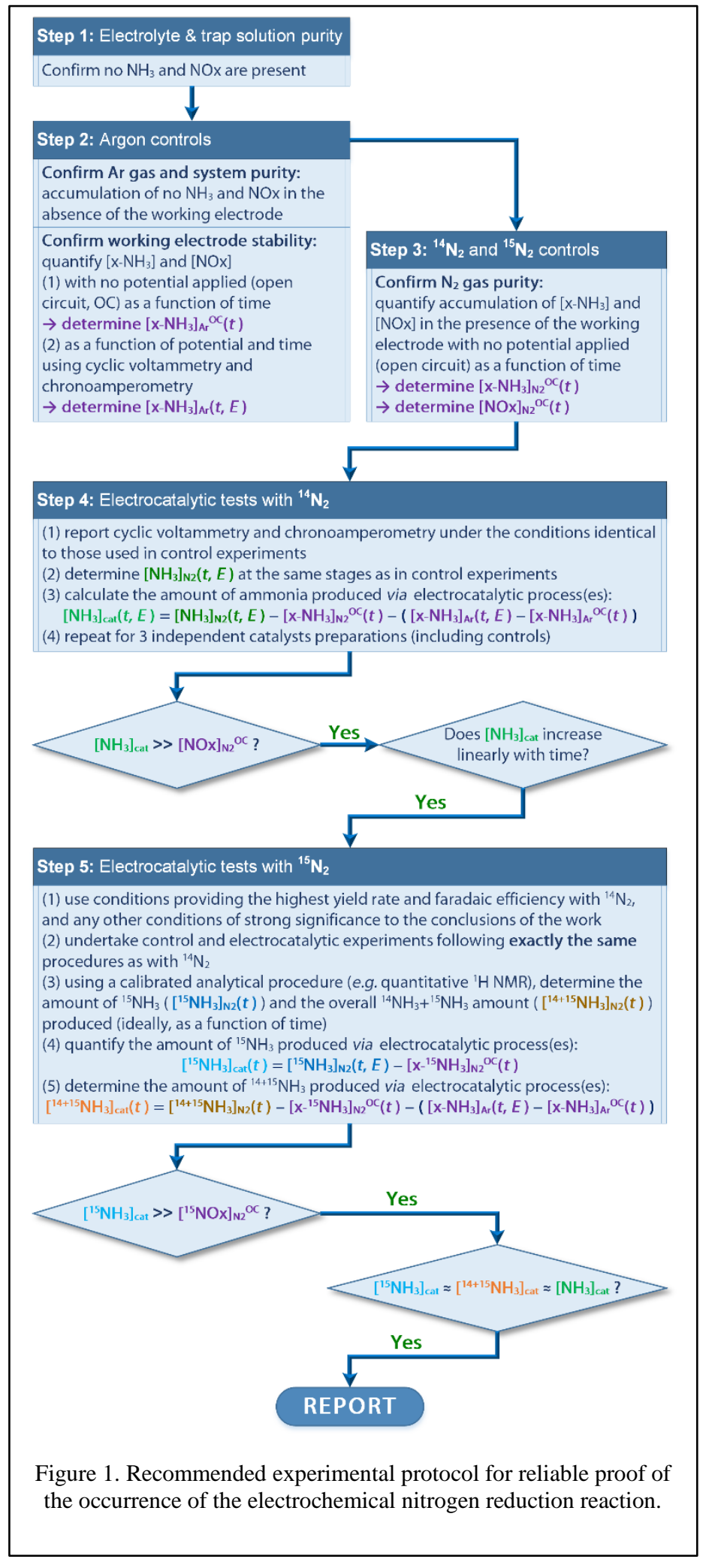

${ }^{15} \mathbf{N}_{2}$-reduction experiments. Headline results should be confirmed quantitatively by a ${ }^{15} \mathrm{~N}_{2}$ reduction experiment to demonstrate that the ammonia produced has originated only from the $\mathrm{N}_{2}$ source. A number of detection methods are available as thoroughly developed in the $\mathrm{N}_{2}$ fixation community. ${ }^{1} \mathrm{H}$ NMR has become commonly used; the characteristic ${ }^{14} \mathrm{NH}_{4}{ }^{+}$triplet, can be used to quantify ammonia separately from ${ }^{15} \mathrm{NH}_{4}{ }^{+}$which is detectable as a strong doublet ${ }^{13,30}$. Quantification is possible either by calibration with ${ }^{15} \mathrm{NH}_{4} \mathrm{Cl}$, or by the use of an internal standard. 
Recognising that background ${ }^{14} \mathrm{~N}_{2}$ and $\mathrm{x}^{-14} \mathrm{NH}_{4}{ }^{+}$are difficult to avoid in this ${ }^{15} \mathrm{NRR}$ experiment, either quantification of both ${ }^{15} \mathrm{NH}_{4}{ }^{+}$and ${ }^{14} \mathrm{NH}_{4}$ is required in the same experiment, or demonstration that ${ }^{15} \mathrm{NH}_{4}{ }^{+}$is within experimental error of total $\mathrm{NH}_{4}{ }^{+}$determined for the same experiment by a routine analysis. The determined $\operatorname{mol}\left({ }^{15} \mathrm{NH}_{4}^{+}\right)$must be substantially greater than $\operatorname{mol}\left({ }^{14} \mathrm{NH}_{4}{ }^{+}\right)$and be quantitatively consistent with the other measurements of NRR yield for this experiment to represent a sufficient proof of NRR.

Time dependent NRR and Nitrogen TON $>1$. It is necessary that an electrocatalyst is capable of continuously producing $\mathrm{NH}_{3}$ through $\mathrm{NRR}$ for an extended period of time ( $>5$ hours) with sustained activity. These experiments should be regarded as mandatory, especially in investigations of the $\mathrm{N}$ containing materials such as metal nitrides and $\mathrm{N}$-doped carbons. Correspondingly, an important goal in the field is to demonstrate TONs well in excess of 1 with respect to any of the species present that could be considered to be reacting in order to indicate genuine long-term capacity of the catalyst.

Reproducibility. Rigorous science always requires reproduction of any observation before it can be reported. However, given the effort-intensity of the protocols detailed above, it may be reasonable to adopt a two-stage approach to experimental design in the NRR field. Stage one might represent a scoping of the behaviour with respect to catalyst compositions, potentials and other significant variables to identify regions of variable-space for deeper study. Stage two represents a detailed investigation within these regions, and data here must be reproduced a minimum of $n=3$ times. It is vital that catalyst preparation is reproduced as well, and at least one data set shown to demonstrate performance of $n=3$ reproductions of a headline catalyst material. Data from stage one can be shown, but must not be drawn into detailed comparisons since its statistical significance is not known.

Data reporting. for the sake of the field it is important that authors present their data in ways that allow easy and direct comparison with other work. A yield rate normalised to the geometric surface area in mol.cm ${ }_{\text {(geom) }}^{-1} \mathrm{~s}^{-1}$ must always be provided. Normalisations based on mass of catalyst or electrochemically active surface area are of fundamental interest, but only as an additional piece of information for mechanistic discussion. The often-overlooked point here is that overall the cost of a practical, large-scale NRR cell, per unit of geometric electrode area, is likely to be a dominant economic factor and therefore it is misleading to focus only on the catalyst mass-yield.

Detection method protocols. Owing to the diverse range of experimental conditions used, a number of detection techniques have been adopted for ammonia detection, each with its own strict conditions that must be adhered to in order to achieve accurate quantification. The techniques most commonly employed are variations on the Nessler or Berthelot reactions ${ }^{31-33}$. However, care must be taken to understand the source and the nature of potential interferences and then design the appropriate controls. The presence of metal ions, various anions and also organic solvents have been shown to interfere with the formation of the chromophores in these methods. It is therefore important that the calibration data must be obtained with exactly the same solution components/concentrations that will be analysed in the NRR experiment. Spiking (i.e. addition of a known amount of ammonia to the solution being analysed) is a very effective way to ensure accuracy. As many solvents interfere with the Berthelot reaction, in non-aqueous NRR studies it is vital that the potential impact of the solvent on the calibration data be investigated and discussed.

Regardless of the method adopted it is strongly recommended that a second confirmatory method be employed to verify headline results. Beyond NMR, ion chromatography is a well-known alternative method with routine limit of detection around $1 \mu \mathrm{M}$. 


\section{Hydrogen versus ammonia - the catalyst conundrum}

Catalysts and mechanisms. Recent development of NRR electrocatalysts has been strongly influenced by the understanding of nitrogenase enzymes that has impressively improved in recent years ${ }^{9}$. Focussing on the metal-complex centres of these enzymes, three distinct types have been identified, Fe-Mo, Fe-Fe and Fe-V, the most commonly studied being Fe-Mo. Around 30\% of the electrocatalysts reported for NRR under ambient conditions are based on Fe and Mo. A range of molecular catalysts have also been investigated, often with a focus on these metals ${ }^{34}$. Broadly, heterogeneous NRR catalysts fall into four main categories: (i) metals, (ii) metal chalcogenides (mainly, oxides and sulphides), (iii) nitrides, and (iv) composites that involve active catalyst centres on a functional support. However, as many recent reports do not include rigorous controls and ${ }^{15} \mathrm{~N}_{2}$ verification, it is difficult at this point to discuss catalyst performance in any comparative depth and space does not permit a fully critical review here. Equally, recent reviews that do not fully address this issue in their survey of the literature are of limited value. A very useful in-depth discussion of catalyst types and mechanisms has been provided recently by Foster et al. ${ }^{9}$ and the interested reader is referred to that review for more detail. Here we provide a brief overview of the understanding of the reaction mechanism and highlight the insights developing from computational studies that we believe are evolving into an important tool for catalyst design.

The reduction of $\mathrm{N}_{2}$ is commonly generalised into the associative and dissociative mechanisms, as shown in the example in Figure $2^{35}$. The main distinction between these is the stage at which the triple bond in the $\mathrm{N}_{2}$ molecule is broken during the reduction steps. In the dissociative mechanism, the $\mathrm{N} \equiv \mathrm{N}$ linkage is reductively broken before the protonation steps initiate, while in the associative mechanism the N-N linkage remains intact throughout some or most of the reduction steps. This associative NRR mechanism is further categorised based on the protonation sequence of the two $\mathrm{N}$ atoms in the $\mathrm{N}_{2}$ molecule, under the assumption that it is adsorbed perpendicular to the catalytic active site $(*)$. However, some density functional theory (DFT) calculations reveal instances where both of the nitrogen atoms can be absorbed to the surface $(* \mathrm{~N}-\mathrm{N} *)$, which is predicted to be a more stable, as well as easily reducible, configuration ${ }^{36}$.

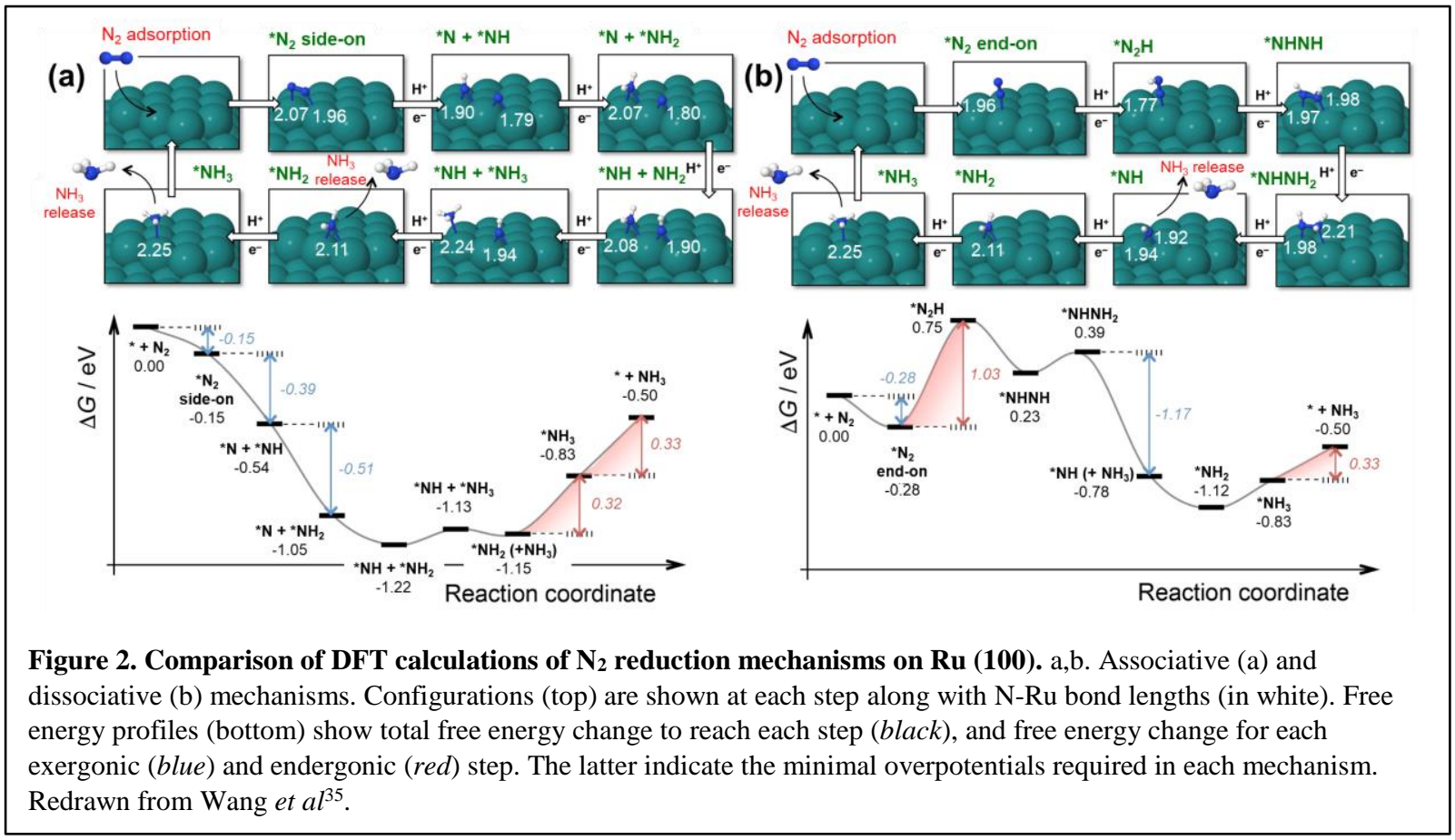

The exact NRR pathway on any given surface remains elusive. The DFT calculations of Skúlason and colleagues ${ }^{36}$ delve into this in a very useful way in an extensive set of calculations that allows comparison of the mechanisms that might theoretically occur on a variety of metals and on flat and 
stepped surfaces; the latter information is particularly informative for the interpretation of the data obtained with various nanostructures. Key results of this study are presented as plots (Figure 3) of the change in the reaction free energy $(\Delta G)$ of the rate limiting processes which can also be expressed as a required minimum overpotential, $U$, as a function of their $\mathrm{N}$ atom adsorption energy $\left(\Delta E_{\mathrm{N}^{*}}\right)$. On both flat and stepped surfaces, the dissociative mechanism is predicted to be an energetically preferable pathway. In the case of the early transition metals (Sc to Fe, and $\mathrm{Y}$ to $\mathrm{Rh}$ ), the analysis suggests that the rate limiting step is one of the protonation reactions, while in the later transition metals $(\mathrm{Co}, \mathrm{Ni}$ and $\mathrm{Pt} / \mathrm{Pd})$ it is the $\mathrm{N}_{2}$ dissociation reaction which is limiting. On the other hand, an associative reaction may be possible on $\mathrm{Fe}, \mathrm{Ru}$ and $\mathrm{Rh}$ and favoured on $\mathrm{Ag}$ and $\mathrm{Au}$. It is worth noting that the reaction on $\mathrm{Au}$ is only predicted to be observable at very negative potentials; this raises questions about the nature of the NRR reported on a variety of Au catalysts.

Skúlason $e t a l{ }^{36}$ also indicate the relatively limited region of potential $v s$. binding energy space where N-binding (green shading in Figure 3 ) is able to compete with the more ubiquitous H-binding for surface coverage of the metal. Combining this surface coverage perspective, in regard to minimising the parallel HER process with the need to minimise the NRR overpotential, the prediction is that $\mathrm{Fe}, \mathrm{W}$, Mo and Ru will be the most effective metal catalysts. More recent DFT studies have provided further detail on the mechanisms for a number of these surfaces ${ }^{37-39}$, as well as other materials including carbides and sulphides ${ }^{40-42}$. A recent study by Wang et al. investigated Ru nano-particles by DFT and experimental studies at low overpotentials, thereby minimising the parallel HER process to achieve an FE of more than $5 \%$ at $-0.01 \mathrm{~V} v s$. reversible hydrogen electrode $(\mathrm{RHE})^{35}$. Developing from that, Suryanto et al. investigated Ru nanoparticles decorated onto $\mathrm{MoS}_{2}$ as a co-catalyst strategy to further lower the HER rate by modulating the conducting properties of the $\mathrm{MoS}_{2}$ through polymorphic control, achieving FE in excess of $17 \%{ }^{42}$; the parallel DFT studies demonstrated the role of nearby surface sites on the $\mathrm{MoS}_{2}$ in providing a supply of absorbed $\mathrm{H}^{\cdot}$ to the $\mathrm{N}_{2}$ molecule absorbed onto the $\mathrm{Ru}$ nanoparticle.

Unfortunately, direct and unambiguous experimental proof of these mechanisms is yet to be obtained. In situ and operando spectroelectrochemical studies of the sort that are enabling progress in other areas of electrocatalysis and electrochemistry are urgently needed. When combined with contemporary theory, these methods can reveal the chemical nature, structure and electronic states of active sites and reaction intermediates. This can aid in understanding the origins of both positive and negative changes in the performance upon modification of a catalyst, or during extended operation. Among the most powerful in situ electrochemical techniques that enable probing both catalyst and surface intermediates are methods using X-rays in different modes ${ }^{43,44}$; useful information on the speciation of adsorbates can be also derived using in situ $\mathrm{IR}^{45}$, and Raman spectroscopy ${ }^{46,47}$. Some of the most recent and truly outstanding developments in the in situ analysis of electrocatalysts are associated with liquid phase transmission electron microscopy that reveals nanometre-scale restructuring of catalysts in action ${ }^{48}$. However, application of many of these techniques to the NRR requires a system where electrochemical conversion of $\mathrm{N}_{2}$ to $\mathrm{NH}_{3}$ is the dominating process and the contribution of the HER is minimal. Even techniques that are chemically specific, for example vibrational spectroscopy and X-ray absorption spectroscopy, may be misleading if the probed process is occurring against a massive background of $\mathrm{H}_{2}$ production wherein surfaces are heavily dominated by absorbed $\mathrm{H}^{-}$-type intermediates. Equally importantly, such studies must strictly comply with the Proof of NRR requirements in order to be meaningful. 


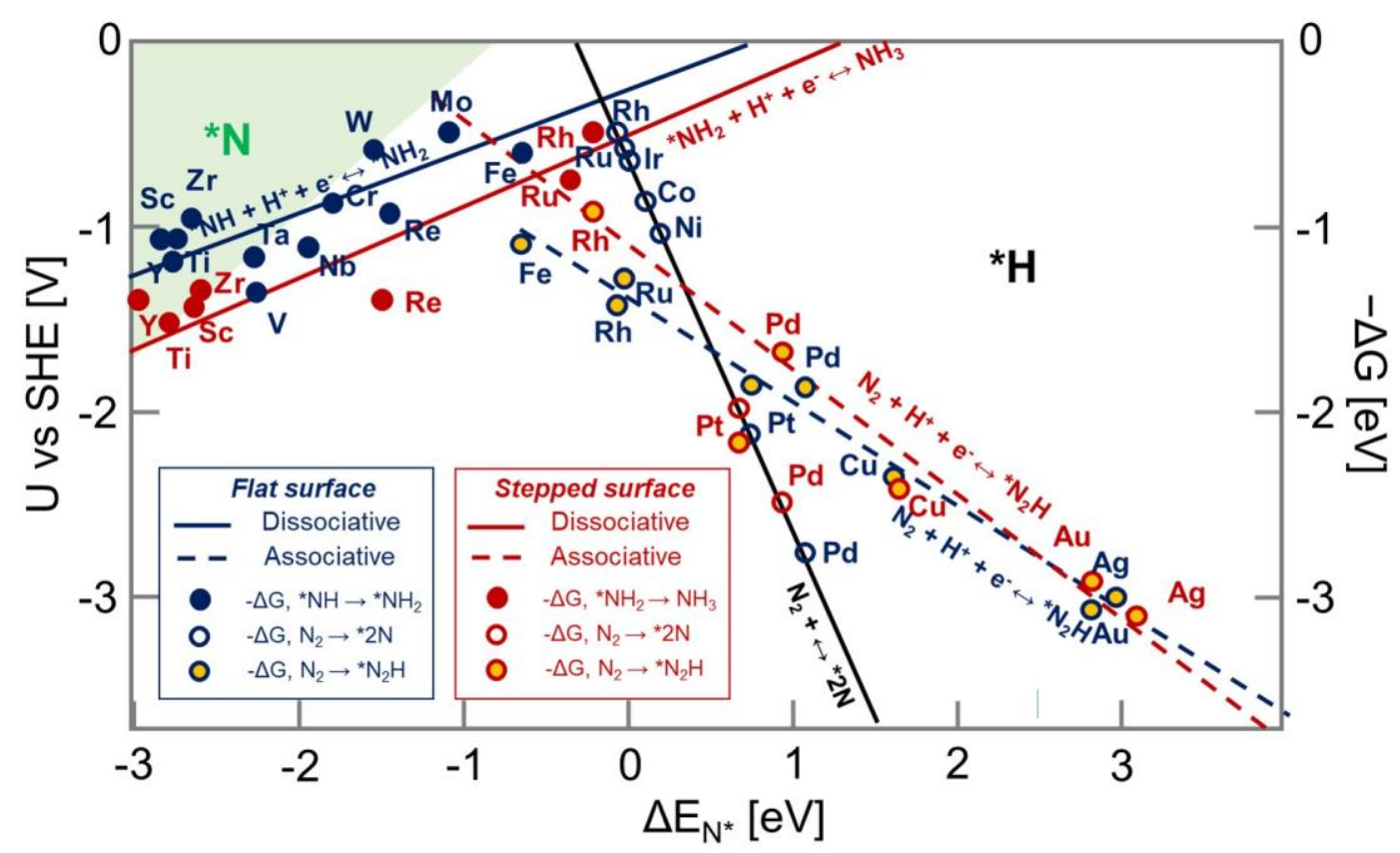

Figure 3. DFT free energy calculations by Skúlason et $\boldsymbol{a l}^{\mathbf{3 6}} \mathrm{Red} / \mathrm{blue}$ lines represent estimates of the overpotentials of the indicated rate limiting reactions on stepped/flat surfaces (black line indicates the situation where the red and black lines overlie one another). Solid lines represent cases where a dissociative reaction is limiting; dashed lines represent rate limiting associative reactions. The area shaded in green indicates the region where the metal surfaces are likely to be occupied by absorbed $\mathrm{N}$; the area in white represents surfaces dominated by $\mathrm{H}$. Redrawn and extended from $\mathrm{ref}^{36}$.

\section{Limiting the hydrogen evolution reaction by supressing proton activity}

To assist an NRR catalyst in attaining high selectively, the availability/activity of the proton can be manipulated to supress the occurrence of the HER. In kinetic terms, NRR is unlikely to be proton concentration limited until some low concentration is reached where the mass transport of protons to the electrode is just sufficient to match the NRR rate. At that point, the faradaic efficiency should reach the highest possible value for a particular system. This kinetic effect is distinct from the standard thermodynamic effect of proton activity that is implicit in the mechanistic discussion above. One approach to control of the supply of proton source involves the use of aprotic electrolytes with a controlled quantity of added water or acid/base. For example, Kim et al. reported the use of ethylenediamine as the electrolysis medium. After background corrections, NRR FE and rates of $17 \%$ and $3.6 \times 10^{-11} \mathrm{~mol} \mathrm{~s}^{-1} \mathrm{~cm}^{-2}$ were observed, significantly more efficient at the time than the generally reported FEs for aqueous media NRR. Our group has also recently reported the application of aprotic ionic liquid (IL) electrolytes for highly efficient $\mathrm{NRR}^{13}$; such electrolytes are widely recognised for their utility in electrochemical applications ${ }^{49}$. A maximum FE of $66 \%$ was obtained on an iron-based cathode in a phosphonium-based IL; this IL was intentionally chosen because it does not contain any nitrogen. DFT calculations reveal that the high FE is the result of a distinctly strong $\mathrm{N}_{2}$ interaction with certain IL anions ${ }^{13}$, leading to high $\mathrm{N}_{2}$ solubility ${ }^{50}$. Further development of these systems is underway to enable advanced operando investigations of the NRR mechanism. However, it is clear that the high viscosity of the IL and the low surface area of the planar electrode employed in this study limited the $\mathrm{NH}_{3}$ formation rate. This was addressed in subsequent work with the use of a less viscous IL mixed with an aprotic solvent and a high-surface area iron-based electrode ${ }^{51}$ enhancing the yield rate by almost an order of magnitude to $2.3 \times 10^{-11} \mathrm{~mol} \mathrm{~s}^{-1} \mathrm{~cm}^{-2}$. Although the $\mathrm{FE}$ was lower in this case (32\%), higher selectivity is expected upon thorough optimisation of a proton activity. Further improvements in the performance are emerging by coupling these engineered electrolytes with other highly active and selective electrocatalysts. 


\section{Nitrides and the Mars-van Krevelen mechanism}

Nitrides are an attractive catalyst concept because of the possibility of dinitrogen activation via the Mars-van Krevelen mechanism operating on such materials. In this mechanism the ammonia is produced from lattice nitride ions and protons, creating lattice vacancies which are subsequently replenished by reductive incorporation of an $\mathrm{N}_{2}$ molecule ${ }^{52}$. Very importantly, nitrides are not expected to be facile HER catalysts. However, two issues emerge in pursuing this direction. Firstly, the experimental Proof of NRR task is considerably more difficult because of the presence of the catalyst nitrogen; this is a case where quantitative demonstration of ${ }^{15} \mathrm{NH}_{3}$ formation, and additionally the incorporation of ${ }^{15} \mathrm{~N}$ into the catalyst structure, is absolutely required as proof of the proposed mechanism. Also, the experiment must demonstrate an extended period of testing; obviously even a small continuous net loss of lattice nitride would be destructive to the catalyst in the long run. Some of the present authors have recently described a rigorous examination of nitrides in the vanadium and niobium families and found both to be inactive towards $\mathrm{N}_{2}$ reduction, despite earlier literature reports to the contrary ${ }^{53}$. This lack of the catalytic activity suggests that the theoretically predicted high rates and selectivity for the NRR might be exclusive to particular crystal facets, which are not abundant on the surfaces of polycrystalline nitrides. Further research in this nitride area might benefit from the design of materials with specifically structured surfaces to mimic the theoretical models. The other aspect of nitride catalysts that needs to be borne in mind is the high reductive potential that is required in some cases to complete the reductive incorporation of $\mathrm{N}_{2}$ into the lattice.

\section{Selectivity, efficiency and rate - the ultimate practical challenges}

The energy cost of NRR is an oft-ignored issue. Recognising that water oxidation is the only viable anode process at large scale, $\sim 1.5 \mathrm{~V} v s$. RHE is required for the oxidative half-reaction. Coupling this to a cathode at $\sim-0.2 \mathrm{~V} v s$. RHE and allowing for $0.1 \mathrm{~V}$ of resistance losses produces a minimum

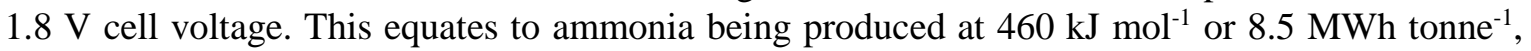
assuming $100 \%$ FE. This is close to the energy cost of the current Haber-Bosch process $(9.5-10.5$ MWh tonne $\left.{ }^{-1}\right)^{54}$. Hence, reductive potentials that are substantially more negative involve an energy input that is likely to be un-competitive in a practical process.

The low selectivity of NRR catalysts exacerbates the energy efficiency problem considerably. A 20\% FE increases the energy cost to $42.5 \mathrm{MWh}^{-1} \mathrm{Mnne}^{-1}$. Some value may be obtained from the $\mathrm{H}_{2}$ byproduct, however this is dependent on a range of factors including scale, and geographic location. It is also unfortunately the case that the NRR is typically carried out at potentials more negative than normal HER (at equivalent partial current densities) and therefore the hydrogen by-product from NRR is produced at a much greater energy cost than in practical water electrolysis.

In this context, the report of high $\mathrm{FE}$ production of $\mathrm{NH}_{3}$ via the reaction of lithium and $\mathrm{N}_{2}$ to form $\mathrm{Li}_{3} \mathrm{~N}$ as a mediator is notable ${ }^{55}$. The $\mathrm{Li}_{3} \mathrm{~N}$ can be hydrolysed to liberate $\mathrm{NH}_{3}$, and the $\mathrm{LiOH}$ byproduct recycled to Li metal in a molten salt mixture at $450^{\circ} \mathrm{C}$ at a minimum cell potential of $\sim 3.0$ $\mathrm{V}$. At this potential the electrical energy cost is $\sim 14 \mathrm{MWh}_{\text {tonne }}{ }^{-1}$.

In gaining a perspective on the broader energy landscape here, it is also important to keep in mind the approach being developed by Wilkinson et al. ${ }^{56}$ at Siemens, based on water electrolysis as a source of sustainable $\mathrm{H}_{2}$ for input to a Haber-Bosch reactor. Their estimates of the energy cost of the whole process is $\sim 13 \mathrm{MWh}$ tonne ${ }^{-1}$ based on practical equipment. This sets an important benchmark for the NRR field, both in terms of energy cost and also ultimate capital cost.

The above discussion has focussed on the nexus between thermodynamics, selectivity and ultimate energy cost. The rate of the process adds a different dimension to the discussion, as it ultimately impacts on the size of the electrode assembly needed to generate a given yield of ammonia. This becomes a major determining factor in the capital cost of the electrolysis cell. For this reason, the yield per unit geometric area of electrode is a practically important property. A number of recent papers have focussed instead only on catalyst mass-normalised yield (without providing the areal yield). The areal density of isolated catalyst atoms/clusters/particles on an electrode support usually 
has a strong effect on catalyst performance and, without a detailed study and discussion of this factor, it is impossible to assess these catalysts in a real-world context.

\section{Prospects and key strategies}

Development of a sustainable ammonia synthesis process for both fertiliser and fuel production is likely to have the same massive global impact in this century as did the Haber-Bosch process 100 years ago. Whether this will involve a direct electrochemical reduction of dinitrogen is dependent on the design and engineering of electrode/catalyst/electrolyte combinations that solve the interlinked, competing, challenges of high selectivity, high energy efficiency and high rate. The complexity of the six-electron, six-proton reduction involved is extreme by traditional electrochemistry standards. Nonetheless, the rapidly developing ability of quantum chemical and other computational techniques to provide accurate detail on the mechanistic steps involved is proving vital in overcoming this complexity. The high selectivity processes described by us and others are also enabling the meaningful application of operando techniques to understand and optimise mechanisms. In particular, catalyst structures, or catalyst-electrolyte combinations, that combine high $\mathrm{N}_{2}$ absorption with reduced rate of direct $\mathrm{H}^{+}$and/or $\mathrm{H}^{\cdot}$ reduction to $\mathrm{H}_{2}$ will provide pathways to high selectivity. For both selectivity and energy efficiency a key strategy should focus on catalyst features, such as defects, edges and steps, that offer low overpotential pathways, i.e. close to or above RHE; theoretical mechanistic studies can be the key to identifying these possibilities, in particular in searching specific surface features that provide low energy mechanisms. Such studies may well provide the pathway to a truly practical process and we enthusiastically commend the theoretical and spectroscopic communities to pursue this important task.

\section{References}

1 Wang, L. et al. Greening Ammonia toward the Solar Ammonia Refinery. Joule 2, 1055-1074 (2018)

2 Gilbert, N. Dirt poor. Nature 483, 525 (2012).

3 Smil, V. Enriching the Earth: Fritz Haber, Carl Bosch, and the Transformation of World Food Production. (MIT Press, 2004).

4 Erisman, J. W., Sutton, M. A., Galloway, J., Klimont, Z. \& Winiwarter, W. How a century of ammonia synthesis changed the world. Nat Geosci. 1, 636 (2008).

5 Service, R. F. Liquid sunshine. Science 361, 120-123 (2018).

6 Shipman, M. A. \& Symes, M. D. Recent progress towards the electrosynthesis of ammonia from sustainable resources. Catal. Today 286, 57-68 (2017).

7 van der Ham, C. J. M., Koper, M. T. M. \& Hetterscheid, D. G. H. Challenges in reduction of dinitrogen by proton and electron transfer. Chem. Soc. Rev. 43, 5183-5191 (2014).

8 Dolan, M. in 2017 AIChE Annual Meeting available from https://publications.csiro.au/rpr/pub?pid=csiro:EP172829 (Minneapolis, 2017).

9 Foster, S. L. et al. Catalysts for nitrogen reduction to ammonia. Nat Catal. 1, 490-500 (2018).

10 Zhan, C.-G., Nichols, J. A. \& Dixon, D. A. Ionization potential, electron affinity, electronegativity, hardness, and electron excitation energy: molecular properties from density functional theory orbital energies. J. Phys. Chem. A 107, 4184-4195 (2003). 
11 Jia, H.-P. \& Quadrelli, E. A. Mechanistic aspects of dinitrogen cleavage and hydrogenation to produce ammonia in catalysis and organometallic chemistry: relevance of metal hydride bonds and dihydrogen. Chem. Soc. Rev. 43, 547-564 (2014).

12 Licht, S. et al. Ammonia synthesis by $\mathrm{N}_{2}$ and steam electrolysis in molten hydroxide suspensions of nanoscale $\mathrm{Fe}_{2} \mathrm{O}_{3}$. Science 345, 637 (2014).

13 Zhou, F. et al. Electro-synthesis of ammonia from nitrogen at ambient temperature and pressure in ionic liquids. Energy Environ. Sci. 10, 2516-2520 (2017).

14 Low, M. J., Ramasubramanian, N. \& Rao, V. S. Reactions of ammonia with porous glass surfaces. J. Phys. Chem. 71, 1726-1734 (1967).

15 Murray, C. A. \& Greytak, T. J. Raman scattering from ammonia adsorbed on the amorphous silica surface. J. Chem. Phys.71, 3355-3365 (1979).

16 Richardson, G., Davies, J. \& Edwards, J. Micromolar ammonia analysis and atmospheric contamination. Fresenius. J. Anal. Chem. 340, 392-394 (1991).

17 Warner, J. X. et al. Increased atmospheric ammonia over the world's major agricultural areas detected from space. Geophys. Res. Lett. 44, 2875-2884 (2017).

18 Ianniello, A. et al. Occurrence of gas phase ammonia in the area of Beijing (China). Atmos. Chem. Phys. 10, 9487-9503 (2010).

19 Boucher, D. L., Davies, J. A., Edwards, J. G. \& Mennad, A. An investigation of the putative photosynthesis of ammonia on iron-doped titania and other metal oxides. J. Photochem. Photobiol. A: Chem. 88, 53-64 (1995).

20 Ritter, S. Cold fusion died 25 years ago, but the research lives on. Scientists continue to study unusual heat-generating effects, some hoping for vindication, others for an eventual payday Chemical and Engineering News 94, 34-39 (2016).

21 Franks, F. Polywater (MIT Press, 1981).

22 Ishibashi, $\mathrm{T}$. et al. $\mathrm{NO}(\mathrm{x})$ contamination in laboratory ware and effect of countermeasures. Nitric Oxide Biol. Chem. 4, 516-525 (2000).

23 Makela, S., Yazdanpanah, M., Adatia, I. \& Ellis, G. Disposable surgical gloves and pasteur (transfer) pipettes as potential sources of contamination in nitrite and nitrate assays. Clinical Chem. 43, 2418-2420 (1997).

24 Greenlee, L. F., Renner, J. N. \& Foster, S. L. The use of controls for consistent and accurate measurements of electrocatalytic ammonia synthesis from dinitrogen. ACS Catal. 8, 78207827 (2018).

25 Cattarin, S. Electrochemical reduction of nitrogen oxyanions in $1 \mathrm{M}$ sodium hydroxide solutions at silver, copper and CuInSe ${ }_{2}$ electrodes. J. Appl. Electrochem. 22, 1077-1081 (1992). 
26 Badea, G. E. Electrocatalytic reduction of nitrate on copper electrode in alkaline solution. Electrochim. Acta 54, 996-1001 (2009).

27 Li, H. 1., Robertson, D. H., Chambers, J. Q. \& Hobbs, D. T. Electrochemical reduction of nitrate and nitrite in concentrated sodium hydroxide at platinum and nickel electrodes. $J$. Electrochem. Soc. 135, 1154-1158 (1988).

28 Ohmori, T., El-Deab, M. S. \& Osawa, M. Electroreduction of nitrate ion to nitrite and ammonia on a gold electrode in acidic and basic sodium and cesium nitrate solutions. $J$. Electroanal. Chem. 470, 46-52 (1999).

29 Chen, J., Yao, B., Li, C. \& Shi, G. An improved Hummers method for eco-friendly synthesis of graphene oxide. Carbon 64, 225-229 (2013).

30 Nazemi, M., Panikkanvalappil, S. R. \& El-Sayed, M. A. Enhancing the rate of electrochemical nitrogen reduction reaction for ammonia synthesis under ambient conditions using hollow gold nanocages. Nano Energy 49, 316-323 (2018).

31 Searle, P. L. The Berthelot or Indophenol Reaction and Its Use in the Analytical Chemistry of Nitrogen Analyst 109, 549 - 568 (1984).

32 T. T. Ngo, A. P. H. P., C. F. Yam, M. Lenhoff. Interference in determination of ammonia with the hypochlorite-alkaline phenol method of Berthelot. Anal. Chem. 52, $46-49$ (1982).

33 Y. Moliner-Martinez, R. H.-H., P. Campins-Falco. Improved detection limit for ammonium/ammonia achieved by Berthelot's reaction by use of solid-phase extraction coupled to diffuse reflectance spectroscopy. Anal. Chim. Acta 534, 327-334 (2005).

34 Roux, Y., Duboc, C. \& Gennari, M. Molecular catalysts for $\mathrm{N}_{2}$ reduction: State of the art, mechanism, and challenges. ChemPhysChem 18, 2606-2617 (2017).

35 Wang, D. et al. Energy-Efficient nitrogen reduction to ammonia at low overpotential in aqueous electrolyte under ambient conditions. ChemSusChem 11, 3416-3422 (2018).

36 Skulason, E. et al. A theoretical evaluation of possible transition metal electro-catalysts for $\mathrm{N}_{2}$ reduction. Phys. Chem. Chem. Phys. 14, 1235-1245 (2012).

37 Qian, J., An, Q., Fortunelli, A., Nielsen, R. J. \& Goddard, W. A. Reaction mechanism and kinetics for ammonia synthesis on the Fe(111) surface. J. Am. Chem. Soc. 140, 6288-6297 (2018).

38 Zhou, F. et al. Electro-synthesis of ammonia from nitrogen at ambient temperature and pressure in ionic liquids. Energy Environ. Sci. 10, 2516-2520 (2017).

39 Liu, C. et al. Theoretical evaluation of possible 2D boron monolayer in $\mathrm{N}_{2}$ electrochemical conversion into ammonia. J. Phys. Chem. C 122, 25268-25273 (2018).

40 Azofra, L. M., Li, N., MacFarlane, D. R. \& Sun, C. Promising prospects for $2 \mathrm{D} \mathrm{d} \mathrm{d}^{2}-\mathrm{d}^{4} \mathrm{M}_{3} \mathrm{C}_{2}$ transition metal carbides (MXenes) in $\mathrm{N}_{2}$ capture and conversion into ammonia. Energy Environ. Sci. 9, 2545-2549 (2016). 
41 Azofra, L. M., Sun, C., Cavallo, L. \& MacFarlane, D. R. Feasibility of $\mathrm{N}_{2}$ binding and reduction to ammonia on Fe-deposited $\mathrm{MoS}_{2} 2 \mathrm{D}$ sheets: A DFT study. Chem. Europ. J. 23, 8275-8279 (2017).

42 Suryanto, B. H. R. et al. $\mathrm{MoS}_{2}$ polymorphic engineering enhances selectivity in the electrochemical reduction of nitrogen to ammonia. ACS Energy Lett. 4, 430-435 (2018).

43 Tesch, M. et al. Evolution of oxygen-metal electron transfer and metal electronic states during Mn-oxide catalyzed water oxidation revealed with in situ soft X-ray spectroscopy. Angew. Chem. doi: doi.org/10.1002/anie.201810825 (2019).

44 Pfeifer, V. et al. The electronic structure of iridium oxide electrodes active in water splitting. Phys. Chem. Chem. Phys. 18, 2292-2296 (2016).

45 Yao, Y., Zhu, S., Wang, H., Li, H. \& Shao, M. A Spectroscopic study on the nitrogen electrochemical reduction reaction on gold and platinum surfaces. J. Am. Chem. Soc. 140, 1496-1501 (2018).

46 Dunwell, M. et al. The central role of bicarbonate in the electrochemical reduction of carbon dioxide on gold. J. Am. Chem. Soc. 139, 3774-3783 (2017).

47 Pavlovic, Z., Ranjan, C., van Gastel, M. \& Schlögl, R. The active site for the water oxidising anodic iridium oxide probed through in situ Raman spectroscopy. Chem. Commun. 53, 12414-12417 (2017).

48 Hodnik, N., Dehm, G. \& Mayrhofer, K. J. J. Importance and Challenges of Electrochemical in Situ Liquid Cell Electron Microscopy for Energy Conversion Research. Acc. Chem. Res. 49, 2015-2022 (2016).

49 MacFarlane, D. R. et al. Ionic liquids and their solid-state analogues as materials for energy generation and storage. Nat Rev.Mater.1, 15005 (2016).

50 Kang, C. S. M., Zhang, X. \& MacFarlane, D. R. Synthesis and Physicochemical Properties of Fluorinated Ionic Liquids with High Nitrogen Gas Solubility. J. Phys. Chem. C 122, 2455024558 (2018).

51 Suryanto, B. H. R. et al. A Rational Electrode-Electrolyte Design for Efficient Ammonia Electrosynthesis under Ambient Conditions. ACS Energy Lett. 3, 1219-1224 (2018).

52 Abghoui, Y. et al. Enabling electrochemical reduction of nitrogen to ammonia at ambient conditions through rational catalyst design. Phys. Chem. Chem. Phys. 17, 4909-4918 (2015).

53 Du, H-L, Gengenbach, T., Hodgetts R, MacFarlane, D.R., Simonov A.N. Critical assessment of the electrocatalytic activity of vanadium and niobium nitrides for the reduction of dinitrogen to ammonia. ACS Sust. Chem. Eng. in review (2019).

54 Rizos, V. Final report: for a study on composition and drivers of energy prices and costs in energy intensive industries: The case of the chemical industry - Ammonia, $<$ https://www.ceps.eu/system/files/Ammonia.pdf> (2014). 
55 McEnaney, J. M. et al. Ammonia synthesis from $\mathrm{N}_{2}$ and $\mathrm{H}_{2} \mathrm{O}$ using a lithium cycling electrification strategy at atmospheric pressure. Energy Environ. Sci. 10, 1621-1630 (2017).

56 Wilkinson, I. Green Ammonia, <https://nh3fuelassociation.org/wpcontent/uploads/2017/11/NH3-Energy-2017-Ian-Wilkinson.pdf> (2017).

\section{Competing interests}

